\title{
Does sputum culture affect the management and/or outcome of community-acquired pneumonia?
}

M.R. Shariatzadeh ${ }^{1}$ and T.J. Marrie ${ }^{2}$

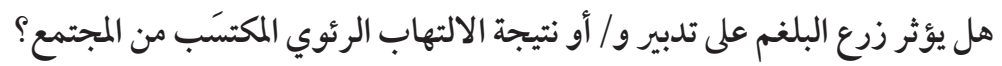

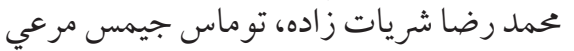

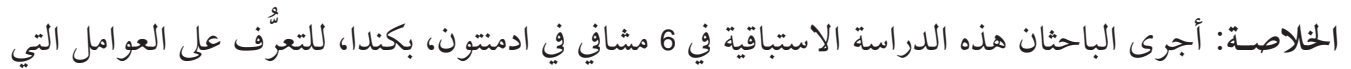

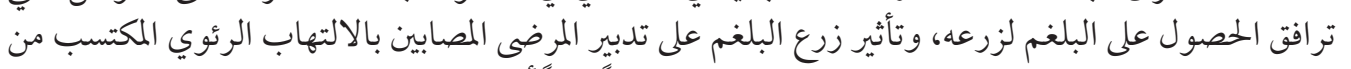

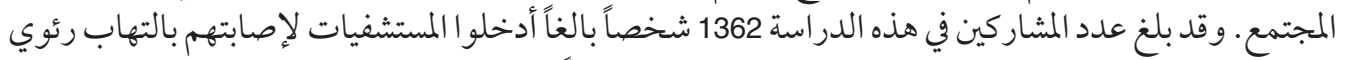

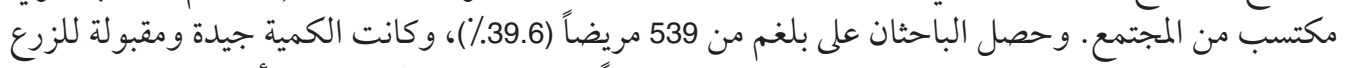

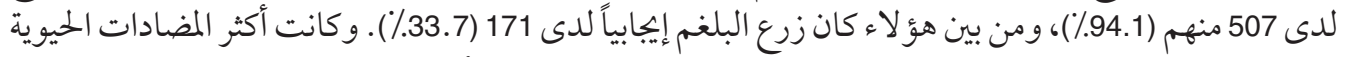

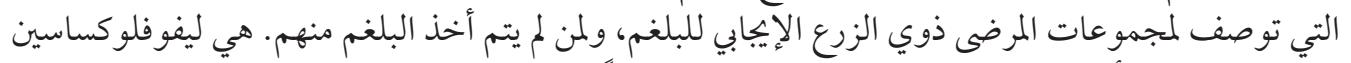

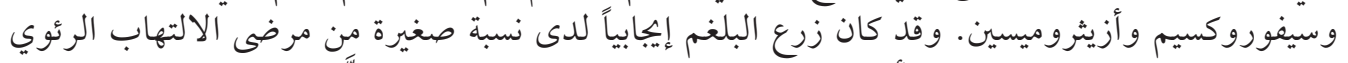

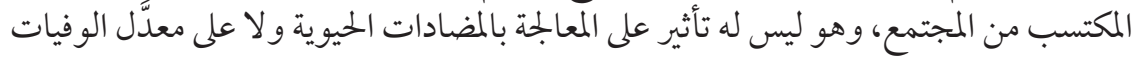

ABSTRACT This prospective study was conducted in 6 hospitals in Edmonton, Canada to determine the factors associated with obtaining sputum for culture and the effect of sputum culture on the management of patients with community-acquired pneumonia (CAP). Participants were 1362 adults who were hospitalized with CAP. Sputum was obtained from 539 (39.6\%) patients, of which 507 $(94.1 \%)$ were good quality, acceptable for culture; 171 (33.7\%) of these had a positive sputum culture. Levofloxacin, cefuroxime and azithromycin were the most common antibiotics prescribed for the groups with positive sputum culture and no sputum collection. Positive sputum culture was demonstrated in only a small number of patients with CAP; this did not affect antimicrobial therapy or mortality.

La culture des expectorations a-t-elle une incidence sur la prise en charge et/ou l'issue de la pneumonie communautaire?

RÉSUMÉ Cette étude prospective a été menée dans six hôpitaux d'Edmonton (Canada) afin de déterminer les facteurs associés à l'obtention d'expectorations à des fins de mise en culture et les effets de celle-ci sur la prise en charge des patients atteints de pneumonie communautaire (PC). 1362 adultes hospitalisés avec une PC ont participé à cette étude. Des expectorations ont été obtenues chez 539 patients (39,6\%), et 507 (94,1\%) d'entre elles étaient d'une qualité satisfaisante et acceptable pour la culture ; pour 171 (33,7\%), cette culture était positive. La lévofloxacine, la céfuroxime et l'azithromycine étaient les antibiotiques les plus prescrits pour les groupes qui avaient une culture positive ou dont les expectorations n'avaient pas été recueillies. La culture des expectorations ne s'est révélée positive que chez un petit nombre de personnes atteintes de PC ; cela n'a pas eu d'incidence sur la thérapie antimicrobienne ou sur la mortalité.

\footnotetext{
${ }^{1}$ Department of Infectious Diseases, Sari University of Medical Sciences, Sari, Mazandaran, Islamic Republic of Iran.

${ }^{2}$ Department of Medicine, University of Alberta, Edmonton, Alberta, Canada (Correspondence to T.J. Marrie: tom.marrie@ualberta.ca).
}

Received: 11/12/06; accepted: 12/04/07 


\section{Introduction}

The value of sputum culture in the diagnosis, management, and outcome of community-acquired pneumonia (CAP) remains a matter of controversy. The Infectious Diseases Society of America pneumonia guidelines recommend Gram staining and culture of expectorated sputum for inpatients with CAP [1]. The reasons for this recommendation are to permit optimal antibiotic selection directed to the causative agent; to limit injudicious antibiotic use in terms of cost; to limit inducible resistance and adverse drug reactions; to support a rational basis for change from parenteral to oral therapy and any change in therapy necessitated by an adverse drug reaction; to identify drug resistant pathogens and monitor trends such as penicillin-resistant Streptococcus pneumoniae, $\beta$-lactamase-producing Haemophilus influenzae, or methicillin-resistant Staphylococcus aureus; and to prompt contact tracing of organisms like $H$. influenzae type $b$ and Neisseria meningitidis [1]. On the other hand, the American Thoracic Society pneumonia guidelines recommend sputum culture only if a drug-resistant pathogen or an organism not covered by usual empiric therapy is suspected [2].

In a recent evaluation of the usefulness of sputum culture for diagnosis of CAP, only $15.8 \%$ of the study population of 1669 patients had a microbiological diagnosis [3]. However, more than $40 \%$ of the patients did not have a sputum specimen collected, and $46 \%$ of the collected specimens were of poor quality and were not cultured [3]. In contrast to this Musher, Montoya and Wanahita argue persuasively for performing sputum culture and indicate that it has a sensitivity of $79 \%$ when bacteraemic pneumococcal pneumonia is used as the gold standard [4]. However, in 47 of the 105 patients in their study, sputum was not submitted or was inadequate for culture.
We conducted this study to evaluate the factors associated with obtaining sputum for culture and to determine the effects of sputum culture on the management and outcome of patients with CAP.

\section{Methods}

The study was carried out from 15 November 2000 to 14 November 2002. It was approved by the research ethics board of the University of Alberta. The study was part of the evaluation of a comprehensive pathway for the treatment of CAP. Participants were adults aged 17 years and older who were hospitalized at any of the 6 hospitals in Capital Health Region, Edmonton, Alberta, Canada with a diagnosis of CAP. Pregnant women, nursing mothers, immunosuppressed patients (patients receiving $\geq 10$ $\mathrm{mg}$ prednisone per day for more than 2 weeks, or other immunosuppressive drugs), and those who had been hospitalized within 30 days prior to the current hospitalization were excluded from the study. Those with HIV infection were excluded if the T-helper lymphocytes CD4 positive count was $<250 / \mathrm{mm}^{3}$. We also excluded patients who were managed as outpatients or needed to be admitted to the intensive care unit, as well as those from acute and sub-acute and continuous care facilities. For patients with multiple admissions, only the first admission was included.

CAP was defined as $\geq 2$ of the following symptoms or signs: fever (temperature $>38^{\circ} \mathrm{C}$ ), cough, sputum production, shortness of breath, pleuritic chest pain, crackles, or consolidation on examination of the chest, together with an acute pulmonary infiltrate evident on the chest radiographs compatible with pneumonia.

"In-patients" are those patients who were hospitalized on the ward and did not need admission to the intensive care unit.

المجلة الصحية لشرق المتوسط، منظمة الصحة العالمية، المجلد الخنامس عشر، العدد ع، 9 +. 
After explaining the procedure to them, all patients were asked, if at all possible, to provide a sputum sample in a sterile sputum specimen container under the supervision of a nurse. Sputum specimens were collected before administering antibiotics in the emergency departments.

Only sputum specimens with $>25$ polymorphonuclear cells and $<10$ epithelial cells per low-power field were considered of good quality and were processed [5]. Sputum specimens were cultured onto sheep blood agar plates incubated in $5 \% \mathrm{CO}_{2}$ for 24 hours. If no growth was evident at 24 hours, plates were further incubated for a total of 48 hours. In addition, the sputum specimen was also cultured onto a chocolate agar plate and a MacConkey agar plate for 24 hours (all media Dylan Inc., Edmonton, Alberta). Microorganisms were identified according to standard methods.

Data collected at the time of enrolment included demographic information, previous history of admission to hospital, drug history, antibiotic therapy before admission, symptoms, laboratory findings and cultures. Severity of illness at the time of admission was evaluated according to the Pneumonia Patient Outcome Research Team (PORT) score [6]. Length of hospital stay was calculated from time of presentation to the emergency department until discharge from hospital.

\section{Statistical analysis}

For analysis of data, SPSS, version 12.0 was used. The significance of proportional differences between nominal variables was determined using the chi-squared test or Fisher exact test, and differences between continuous variables were determined using the Student $t$-test. A 2 -tailed $P<0.05$ was used to define statistical significance. Logistic regression was performed using those variables found significant in univariate analysis.

\section{Results}

The study population of 1362 consecutive patients with bacterial CAP comprised 722 men (53\%) and 640 women (47\%), ranging in age from 18.0 years to 104.8 years, mean 69.0 [standard deviation (SD) 18.4] years. Sputum was collected from 539 (39.6\%) patients; 507 (94.1\%) samples were acceptable for processing for culture. We recovered $\geq 1$ microorganism from 171 (33.7\%) specimens (representing $12.6 \%$ of the initial 1362 patients).

Isolated microorganisms from sputum included Strep. pneumoniae $(n=59), H$. influenzae $(n=40)$, Staph. aureus $(n=23)$, Moraxella catarrhalis $(n=15)$, Pseudomonas aeruginosa $(n=14)$, Strep. spp. $(n=12)$, Klebsiella pneumoniae $(n=5)$, $H$. parainfluenzae $(n=4)$, Stenotrophomonas (Xanthomonas) maltophilia $(n=3)$, Escherichia coli $(n=3)$, Serratia marcescens $(n=2)$, Acinetobacter calcoaeticus $(n=2)$, Pseudomonas fluorescens $(n=2)$, Alcaligenes xylosoxidans $(n=1)$, Proteus mirabilis $(n=1)$, Enterobacter cloacae $(n$ $=1)$ and Klebsiella ozaenae $(n=1)$. There were no isolates of methicillin-resistant Staph. aureus.

Female sex, heart disease, dementia, altered mental status, risk classes IV-V [6] and death as an outcome during hospitalization were all associated with not having sputum collected for culture. Logistic regression analysis of variables significant in the univariate analysis (Table 1) showed sputum collection was dependent on site of hospitalization - site D versus site $\mathrm{C}$ $(P<0.001)$, and site $\mathrm{C}$ versus sites $\mathrm{E}(P$ $=0.045)$ and $\mathrm{F}(P=0.001)$; also male versus female $(P=0.023)$; smokers versus non-smokers $(P=0.017)$; in those with cough versus no cough $(P<0.001)$; and no altered mental state versus altered mental state $(P=0.001)$. 


\begin{tabular}{|c|c|c|c|c|c|}
\hline \multirow[t]{2}{*}{ Characteristic } & \multicolumn{2}{|c|}{$\begin{array}{l}\text { Patients with } \\
\text { positive sputum } \\
\text { culture } \\
(n=171)\end{array}$} & \multicolumn{2}{|c|}{$\begin{array}{l}\text { Patients with no } \\
\text { sputum collection } \\
\qquad(n=823)\end{array}$} & \multirow[t]{2}{*}{$P$-value } \\
\hline & & & No. & $\%$ & \\
\hline \multicolumn{6}{|l|}{$\overline{S e x}$} \\
\hline Male & 97 & 56.7 & 412 & 50.1 & 0.113 \\
\hline Female & 74 & 43.3 & 411 & 49.9 & \\
\hline \multicolumn{6}{|l|}{ Smoking status } \\
\hline Smoker & 46 & 26.9 & 179 & 21.7 & 0.002 \\
\hline Ex-smoker & 69 & 40.4 & 254 & 30.9 & \\
\hline Non-smoker & 36 & 21.1 & 278 & 33.8 & \\
\hline Not documented & 20 & 11.7 & 112 & 13.6 & \\
\hline \multicolumn{6}{|l|}{ Symptom } \\
\hline Fever & 87 & 50.9 & 345 & 41.9 & 0.032 \\
\hline Shaking chills & 51 & 29.8 & 165 & 20.0 & 0.005 \\
\hline Shortness of breath & 121 & 70.8 & 509 & 61.8 & 0.028 \\
\hline Cough & 150 & 87.7 & 512 & 62.2 & $<0.001$ \\
\hline Altered mental state & 13 & 7.6 & 146 & 17.7 & 0.001 \\
\hline \multicolumn{6}{|l|}{ Site, hospital } \\
\hline A & 45 & 26.3 & 113 & 13.7 & $<0.001$ \\
\hline$B$ & 38 & 22.2 & 238 & 28.9 & \\
\hline C & 34 & 19.9 & 132 & 16.0 & \\
\hline D & 33 & 19.3 & 130 & 15.8 & \\
\hline$E$ & 13 & 7.6 & 133 & 16.2 & \\
\hline $\mathrm{F}$ & 8 & 4.7 & 77 & 9.4 & \\
\hline \multicolumn{6}{|l|}{ Risk class ${ }^{a}$} \\
\hline I & 2 & 1.2 & 11 & 1.3 & 0.178 \\
\hline II & 43 & 25.1 & 140 & 17.0 & \\
\hline III & 28 & 16.4 & 147 & 17.9 & \\
\hline IV & 61 & 35.7 & 320 & 38.9 & \\
\hline V & 37 & 21.6 & 205 & 24.9 & \\
\hline \multicolumn{6}{|l|}{ Mortality } \\
\hline Died & 9 & 5.3 & 114 & 13.9 & 0.002 \\
\hline Alive & 162 & 94.7 & 709 & 86.1 & \\
\hline
\end{tabular}

a Source: reference [6].

There were no significant differences between patients with positive sputum culture and those with no sputum collection regarding sex, risk class and co-morbidity (including asthma, chronic obstructive pulmonary disease, diabetes mellitus, heart dis- ease and cancer). However the mean age of the sputum positive group, 65.8 (SD 19.1) years, was lower than mean age of those who did not have a sputum culture done, 70.9 (SD 18.1) years $(P=0.001)$. Patients with positive sputum culture were more

المجلة الصحية لشرق المتوسط، منظمة الصحة العالمية، المجلد الخامس عشر، العدد ع، 9 . ب 
likely to have fever, shaking chills, shortness of breath and cough, and less likely to have altered mental state than patients with no sputum collection (Table 1).

Table 2 shows sputum collection, quality and culture results according to the PORT score in 1362 patients with CAP. The percentage of patients with sputum collected decreased with increasing risk class, and since age is the major driver of risk class, this is likely to be a reflection of increasing age.

Levofloxacin ( $n=137,80.1 \%)$ cefuroxime $(n=37,21.6 \%)$ and azithromycin $(n$ $=32,18.7 \%$ ) were the most common antibiotics prescribed for those with positive sputum culture; in the group from whom no sputum was collected, corresponding values were: levofloxacin $677(82.3 \%)$, cefuroxime $172(20.9 \%)$ and azithromycin $130(15.8 \%)$. Patients with a positive sputum culture were more likely to receive ciprofloxacin and ceftazidime as part of their antibiotic regimen. Of the 46 patients who gave no sputum sample and who received ciprofloxacin or ceftazidime, 9 died compared to 3 of the 27 with a positive sputum culture who received these 2 drugs $(P=0.51)$. Ninetythree $(54.4 \%)$ patients with positive sputum culture and $473(57.5 \%)$ of those from whom no sputum was collected received monotherapy $(P=0.497)$.

The mean length of stay was statistically significantly lower in the positive sputum culture group, 8.8 (SD 8.0) days than in the group from whom no sputum was collected, 10.8 (SD 13.9) days $(P=0.012)$. Patients with positive sputum culture had lower death rate $(9 / 171,5.3 \%)$ both in comparison with patients from whom no sputum was collected $(114 / 823,13.9 \%)(P=0.002)$ and patients with "negative or poor or no sputum collection" $(136 / 1191,11.4 \%)(P$ $=0.015)$. However on logistic regression analysis these differences disappeared.

Table 3 shows the results of sputum culture done on those who had no prior antibiotic treatment versus those who had received antibiotics prior to hospitalization. The number of specimens with Staph. aureus, aerobic Gram-negative rods and fungi was higher in latter group

\section{Discussion}

For decades, sputum culture as a tool for the management of CAP has been a matter of controversy [5,7]. Although sputum culture is considered the basis for the bacteriological diagnosis of pneumonia, its reliability has been challenged because of low sensitivity and specificity [8]. Most of the time sputum specimens submitted for culture were not collected correctly and contained oropharyngeal or upper respiratory secretions [8]. However in a prospective study of the diagnostic value of sputum culture in acute pneumonia in 16 patients, culture of transtracheal aspirate and/or bronchial aspi-

\begin{tabular}{|c|c|c|c|c|c|c|c|c|c|}
\hline & & \multicolumn{2}{|c|}{ Risk class III } & \multicolumn{2}{|c|}{ Risk class IV, V } & \multicolumn{2}{|c|}{ Total } & \multirow[t]{2}{*}{$P$-value } \\
\hline & \multicolumn{2}{|c|}{$\begin{array}{l}\text { Table } 2 \text { Sputum collection, quality and } \\
\text { Outcome Research Team class in } 1362 \\
\text { Characteristic } \\
\begin{array}{ccc}\text { Risk class I, II } \\
\text { n/N } \%\end{array} \\
\end{array}$} & $\mathrm{n} / \mathrm{N}$ & $\%$ & $\mathrm{n} / \mathrm{N}$ & $\%$ & $n / N$ & $\%$ & \\
\hline Sputum collection done & $131 / 282$ & 46.5 & $102 / 249$ & 41.0 & $306 / 831$ & 36.8 & $539 / 1362$ & 39.6 & 0.015 \\
\hline Good quality sputum & $122 / 131$ & 87.1 & $93 / 102$ & 91.2 & $292 / 306$ & 98.3 & $507 / 539$ & 94.1 & $<0.001$ \\
\hline $\begin{array}{l}\text { Organism isolated from } \\
\text { sputum culture }\end{array}$ & $45 / 122$ & 36.9 & $28 / 93$ & 30.1 & 98/292 & 33.6 & $171 / 507$ & 33.7 & 0.579 \\
\hline
\end{tabular}




\begin{tabular}{|c|c|c|c|c|}
\hline \multirow[t]{2}{*}{ Organism recovered } & \multicolumn{2}{|c|}{$\begin{array}{l}\text { No prior antibiotic } \\
\text { treatment }(n=81)\end{array}$} & \multicolumn{2}{|c|}{$\begin{array}{c}\text { Prior antibiotic } \\
\text { treatment }(n=90)\end{array}$} \\
\hline & No. & $\%$ & No. & $\%$ \\
\hline Streptococcus pneumoniae & 35 & 43.2 & 24 & 26.7 \\
\hline Haemophilus influenzae & 25 & 30.9 & 15 & 16.7 \\
\hline Moraxella catarrhalis & 9 & 11.1 & 6 & 6.7 \\
\hline Pseudomonas aeruginosa & 8 & 9.9 & 6 & 6.7 \\
\hline Staphylococcus aureus & 7 & 8.6 & 16 & 17.8 \\
\hline Haemophilus parainfluenzae & 1 & 1.2 & 3 & 3.3 \\
\hline Pseudomonas fluorescens & 1 & 1.2 & 1 & 1.1 \\
\hline Klebsiella pneumoniae & 1 & 1.2 & 4 & 4.4 \\
\hline Serratia marcescens & 1 & 1.2 & 1 & 1.1 \\
\hline Streptococcus pyogenes & 1 & 1.2 & 7 & 7.8 \\
\hline Streptococcus agalactiae & 0 & - & 3 & 3.3 \\
\hline Stenotrophomonas maltophilia & 0 & - & 3 & 3.3 \\
\hline Escherichia coli & 0 & - & 3 & 3.3 \\
\hline Acinetobacter calcoaceticus & 0 & - & 2 & 2.2 \\
\hline Streptococcus anginosus & 0 & - & 1 & 1.1 \\
\hline Klebsiella ozaenae & 0 & - & 1 & 1.1 \\
\hline Alcaligenes xylosoxidans & 0 & - & 1 & 1.1 \\
\hline Enterobacter cloacae & 0 & - & 1 & 1.1 \\
\hline Proteus mirabilis & 0 & - & 1 & 1.1 \\
\hline Fungal isolate & 4 & 4.9 & 25 & 27.7 \\
\hline Total (excluding fungal isolates) ${ }^{a}$ & 89 & - & 99 & - \\
\hline
\end{tabular}

aln some samples more than one organism was isolated.

rate from patients with acute pneumonia did not provide a diagnosis any more frequently than from sputum culture alone [5]. In our study, sputum was collected from only $40 \%$ of cases, however, $94 \%$ of the collected sputum samples were of good quality.

Although one-third of the good quality sputum specimens yielded a causative organism, we actually isolated microorganisms from only $12.6 \%$ of the patients with CAP. This compares with a prospective study in 2005 of 262 patients hospitalized with CAP, of 157 patients who had sputum collected, only $44(28.0 \%)$ were adequate for culture; 36 of these gave a positive result, which meant that $13.7 \%$ of all patients in the study had a positive culture [9].
In a cohort study of 1669 patients for assessment of the usefulness of sputum culture for diagnosis of CAP, only $532(54 \%)$ of the 983 patients with sputum production gave a good quality sample, and overall $264(15.8 \%)$ patients had positive sputum culture [3]. There were no significant differences in rates for sputum sample obtained, good quality sample and positive sputum culture according to severity of pneumonia using the PORT severity of illness scoring system. However, in our study we noted a higher rate of sputum collection for low risk classes I-II compared to high risk classes IV-V; but there were more good quality sputum samples in classes IV-V than in classes I-II and III. 
In a study of sputum examination in the management of patients with CAP in a primary care hospital, $36.4 \%$ of 116 patients were capable of producing a sputum sample, of which $54.7 \%$ gave samples valid for culture [10]. There was a delay of more than 24 hours in $31 \%$ of the patients for collection of sputum and in 52\% for processing. Delay in processing was associated with an increase in the isolation of Candida spp. In a number of studies [10-13], including ours, microorganism recovery was very low in sputum cultures owing to factors such as processing of sputum collection and prior antibiotic therapy, which can decrease diagnostic yield. The value of sputum culture is decreased by the administration of antibiotics before hospitalization, however in a substantial number of samples, Strep. pneumoniae and $H$. influenzae were isolated in our patients even though they had taken antibiotics before admission. In a previous study, it was noted that increasing prevalence of Gram-negative bacilli among oropharyngeal bacterial flora in patients with illnesses of varying severity was not correlated with antibiotic administration or inhalation therapy; in our study, however, we found an increased number of Gramnegative rods in patients who had received antibiotics before admission [14].
Patients with a positive sputum culture for aerobic Gram-negative bacteria were more likely to receive ciprofloxacin and ceftazidime as part of their antibiotic regimen. However, use of these antibiotics did not explain the trend towards reduced mortality.

The major limitation of the present study is that we did not have sputum specimens from all the patients.

To sum up, sputum culture was performed infrequently and a positive sputum culture did not influence mortality rate or length of stay.

\section{Acknowledgements}

This study was funded by an independent research establishment grant from Alberta Heritage Foundation for Medical Research and by grants in aid from Capital Health, Abbott Canada, Pfizer Canada, and JannsenOrtho Canada.

We thank the following CAP pathway nurses: JoAnne de Jager, Linda Gardner, Lynne Korobanik, Tammy Pfeiffer, Cynthia Proskow, Sue Marshall, Nancy Baker, Nan Horne and Fredrika Herbert. The staff of EPICORE carried out data management for the project and Jane Huang helped with data analysis.

\section{References}

1. Bartlett JG et al. Practice guidelines for the management of community-acquired pneumonia in adults. Clinical infectious diseases, 2000, 31(2):347-82.

2. Niederman MS et al, American Thoracic Society. Guidelines for the management of adults with community-acquired pneumonia. Diagnosis, assessment of severity, antimicrobial therapy, and prevention. American journal of respiratory and critical care medicine, 2001, 163(7):173054.
3. Garcia-Vazquez E et al. Assessment of the usefulness of sputum culture for diagnosis of community-acquired pneumonia using the PORT predictive scoring system. Archives of internal medicine, 2004, 164(16):1807-11.

4. Musher DM, Montoya R, Wanahita A. Diagnostic value of microscopic examination of Gram-stained sputum and sputum cultures in patients with bacteremic pneumococcal pneumonia. Clinical infectious diseases, 2004, 39(2):165-9. 
5. Thorsteinsson BM, Musher DM, Fagan T. The diagnostic value of sputum culture in acute pneumonia. Journal of the American Medical Association, 1975, 233(8):894-5.

6. Fine $\mathrm{M} \mathrm{J}$ et al. A prediction rule to identify low-risk patients with community -acquired pneumonia. New England journal of medicine, 1997, 336(4):243-50.

7. Barrett-Connor E. The nonvalue of sputum culture in the diagnosis of pneumococcal pneumonia. American review of respiratory disease, 1971, 103(6):845-8.

8. Plouffe JF, McNally C, File TM Jr. Value of noninvasive studies in communityacquired pneumonia. Infectious disease clinics of North America, 1998, 12(3):68999.

9. Van der Eerden MM et al. Value of intensive diagnostic microbiological investigation in low- and high-risk patients with community-acquired pneumonia. European journal of clinical microbiology \& infectious diseases, 2005, 24(4):241-9.
10. Ewig $S$ et al. Applying sputum as a diagnostic tool in pneumonia -limited yield, minimal impact on treatment decisions. Chest, 2002, 121(5):1486-92.

11. Rello $\mathrm{J}$ et al. Microbiological testing and outcome of patients with severe community-acquired pneumonia. Chest, 2003, 123(1):174-80.

12. Meehan TP et al. Quality of care, process, and outcomes in elderly patients with pneumonia. Journal of the American Medical Association, 1997, 278(23):2080-4.

13. Ruiz M et al. Severe community-acquired pneumonia. Risk factors and follow-up epidemiology. America journal of respiratory and critical care medicine, 1999, 160(3):923-9.

14. Johanson WG, Pierce AK, Sanford JP. Changing pharyngeal bacterial flora of hospitalized patients. Emergence of gram-negative bacilli. New England journal of medicine, 1969, 281(21):1137-40. 